\title{
Simple Carbohydrate Restriction Could Bring about the Renoprotective Effect of Sodium-Glucose Cotransporter 2 Inhibitors in Diabetes Treatment
}

\section{Yuji Aoki*}

Matsumoto University Graduate School of Health Science, Japan

*Corresponding Author: Yuji Aoki, Matsumoto University Graduate School of Health Science, Japan.

Received: July 12, 2019; Published: July 26, 2019

DOI: $10.31080 /$ ASNH.2019.03.0384

\begin{abstract}
Recent major clinical trials have revealed that sodium-glucose cotransporter 2 (SGLT2) inhibitors were effective on heart failure and renal disease progression in patients with type 2 diabetes. SGLT2 inhibitors decrease hyperglycemia independently of insulin by increasing urinary glucose excretion through the inhibition of SGLT2 for glucose reabsorption in the proximal renal tubule. It has been suggested that excessive intake of carbohydrates was associated with obesity and chronic kidney disease. It is expected that carbohydrate restriction can bring about the renoprotective effect of SGLT2 inhibitors in diabetes treatment. Carbohydrate-rich foods contain dietary fiber and micronutrients. Considering also that it takes time to change people's lifestyles, it would be wise to implement proper restriction of carbohydrates or simple carbohydrates while using SGLT2 inhibitors timely and effectively.
\end{abstract}

Keywords: Sodium-Glucose Cotransporter 2 Inhibitor; Type 2 Diabetes; Obesity; Chronic Kidney Disease; Carbohydrate; Lifestyle

\section{Clinical trials with SGLT2 inhibitors}

Previous major trials $[1,2]$ in patients with type 2 diabetes indicated concern that use of certain glucose-lowering drugs was associated with adverse cardiovascular outcomes. Recent similar trials [3-6] with dipeptidyl peptide 4 inhibitors, a new class of antidiabetic drugs, have demonstrated non-inferiority for cardiovascular outcomes, but repeatedly suggesting no further beneficial effects on cardiovascular outcomes by tightly improving glycemic control. Notably, it was reported at the 79th scientific sessions of American Diabetes Association (June 7-11, 2019) that a dipeptidyl peptidase 4 inhibitor of linagliptin and a modern sulfonylurea of glimepiride had similar cardiovascular safety profiles, whereas glimepiride was associated with a higher risk for hypoglycemia than linagliptin (the CAROLINA (Cardiovascular Outcome Trial of Linagliptin versus Glimepiride in Type 2 Diabetes) trial).

In this line, it was surprising that the EMPA-REG OUTCOME (Empagliflozin Cardiovascular Outcome Event Trial in T2DM Patients) study showed that one of sodium-glucose cotransporter 2 (SGLT2) inhibitors, another new class of antidiabetic drugs, had beneficial effects on cardiovascular morbidity and mortality in patients with type 2 diabetes at high risk for cardiovascular events [7]. The following Canagliflozin Cardiovascular Assessment Study (CANVAS) trial demonstrated that such results on cardiovascular outcomes were attributable to a class effect of SGLT2 inhibitors [8]. The latest trial with an SGLT2 inhibitor, the Dapagliflozin Effect on Cardiovascular Events-Thrombolysis in Myocardial Infarction 58 (DECLARE-TIMI 58) trial, has confirmed that SGLT2 inhibitors were effective especially on heart failure and renal disease progression in a broad population of patients with type 2 diabetes [9].

\section{Renoprotective effect of SGLT2 inhibitors}

SGLT2 inhibitors decrease hyperglycemia independently of insulin by increasing urinary glucose excretion through the inhibition of SGLT2 for glucose reabsorption in the proximal renal tubule. They have some advantages including modest weight loss, low risk of hypoglycemia and mild decrease of blood pressure [10]. It is suggested that the mechanisms behind the beneficial effects of SGLT2 inhibitors on heart failure and renal disease progression include osmotic diuresis, reductions in arterial stiffness and the rate pres- 
sure product, and direct renovascular effects through activating tubuloglomerular feedback [11,12]. As for the renoprotective effect of SGLT2 inhibitors, proximal tubulopathy [13] needs to be taken into consideration in addition to impaired tubuloglomerular feedback (or single nephron hyperfiltration) [14]. It was demonstrated that SGLT2 inhibitors did not prevent the decline of glomerular filtration rate in the rat remnant kidney model under a normoglycemic state [15].

Proximal tubular cells require high energy for their enormous reabsorptive functions mainly driven by sodium-potassium ATPase, rendering them susceptible to hypoxia and oxidative stress $[13,16]$. SGLT2 inhibitors are supposed to save the energy for the reabsorptive functions, leading to protection against proximal tubulopathy. Elevation of the hematocrit during SGLT2 inhibitor therapy is presumed to enhancement of erythropoiesis by the recovery from tubulointerstitial hypoxia in addition to hemoconcentration [17]. We have hypothesized that vitamin C reabsorbed through sodium-vitamin $C$ cotransporter 1 , which is inhibited in a hyperglycemic state and restored by SGLT2 inhibitors, contributes to the renoprotective effect as an antioxidant [18].

\section{SGLT2 inhibitors versus carbohydrate restriction}

The beneficial effects of SGLT2 inhibitors in diabetes treatment recall the STOP-Noninsulin-Dependent Diabetes Mellitus (NIDDM) trial [19], in which the treatment with acarbose, an $\alpha$-glucosidase inhibitor, reduced the risk of cardiovascular disease and hypertension in patients with impaired glucose tolerance. These seem to imply that an excessive amount of glucose in the body is rather harmful and should be excreted in the urine or feces (for bacterial fermentation). Familial renal glucosuria with normoglycemia is often referred to as a natural model for SGLT2 inhibition, indicating activation of the renin-angiotensin-aldosterone system without an unfavorable prognosis [20,21]. At least, renal glucosuria with normolgycemia is not regarded as an advantage.

Is the effect of SGLT2 inhibitors equivalent to that of carbohydrate restriction in diabetes treatment? A clinical report demonstrated that a low carbohydrate diet was effective in improving renal function in obese or diabetic patients with chronic kidney disease [22]. It has been shown that excessive intake of carbohydrates or simple carbohydrates was associated with obesity and chronic kidney disease [23-25]. It is therefore expected that carbohydrate restriction can bring about the renoprotective effect of SGLT2 inhibitors in obesity or diabetes treatment, where renal tubules are overloaded with an excessive amount of glucose. Carbohydrate-rich foods contain dietary fiber and micronutrients. Considering also that it takes time to change people's lifestyles, it would be wise to implement proper restriction of carbohydrates or simple carbohydrates while using SGLT2 inhibitors timely and effectively in diabetes treatment.

\section{Conclusion}

Recent major clinical trials have demonstrated that SGLT2 inhibitors have a renoprotective effect in patients with type 2 diabetes. It is inferred from the relevant literature that an excessive amount of glucose can cause proximal tubulopathy or chronic kidney disease. Therefore, carbohydrate or simple carbohydrate restriction could also bring about such a renoprotective effect. A more proper and effective combination of simple carbohydrate restriction and use of SGLT2 inhibitors needs to be explored in diabetes treatment, considering dietary fiber and micronutrients in carbohydrates and glucosuria itself probably as a disadvantage.

\section{Conflict of Interest}

The author has indicated no potential conflict of interest.

\section{Bibliography}

1. The Action to Control Cardiovascular Risk in Diabetes Study Group. "Effects of intensive glucose lowering in type 2 diabetes". The New England Journal of Medicine 358.24 (2008): 2545-2559.

2. The ADVANCE Collaborative Group. "Intensive blood glucose control and vascular outcomes in patients with type 2 diabetes". The New England Journal of Medicine 358.24 (2008): 2560-2572.

3. Scirica BM., et al. "Saxagliptin and cardiovascular outcomes in patients with type 2 diabetes mellitus". The New England Journal of Medicine 369.14 (2013): 1317-1326.

4. White WB., et al. "Alogliptin after acute coronary syndrome in patients with type 2 diabetes". The New England Journal of Medicine 369.14 (2013): 1327-1335.

5. Green JB., et al. "Effect of sitagliptin on cardiovascular outcomes in type 2 diabetes". The New England Journal of Medicine 373.3 (2015): 232-242. 
6. Rosenstock J., et al. "Effect of linagliptin vs placebo on major cardiovascular events in adults with type 2 diabetes and high cardiovascular and renal risk: The CARMELINA randomized clinical trial". The Journal of the American Medical Association 321.1 (2019): 69-79.

7. Zinman B., et al. "Empagliflozin, cardiovascular outcomes, and mortality in type 2 diabetes". The New England Journal of Medicine 373.22 (2015): 2117-2128.

8. Neal B., et al. "Canagliflozin and cardiovascular and renal events in type 2 diabetes". The New England Journal of Medicine 377.7 (2017): 644-657.

9. Wiviott SD., et al. "Dapagliflozin and cardiovascular outcomes in type 2 diabetes". The New England Journal of Medicine 380.4 (2019): 347-357.

10. Mikhail N. "Place of sodium-glucose co-transporter type 2 inhibitors for treatment of type 2 diabetes". World Journal of Diabetes 5.6 (2014): 854-859.

11. Fitchett D., et al. "Heart failure outcomes with empagliflozin in patients with type 2 diabetes at high cardiovascular risk: results of the EMPA-REG OUTCOME trial". European Heart Journal 37.19 (2016): 1526-1534.

12. Wanner C., et al. "Empagliflozin and progression of kidney disease in type 2 diabetes". The New England Journal of Medicine 375.18 (2016): 323-334.

13. Gilbert RE. "Proximal tubulopathy: prime mover and key therapeutic target in diabetic kidney disease". Diabetes 66.4 (2017): 791-800.

14. Fioretto P., et al. "SGLT2 inhibitors and the diabetic kidney". Diabetes Care 39.S2 (2016): S165-S171.

15. Zhang Y., et al. "Sodium-glucose linked cotransporter-2 inhibition does not attenuate disease progression in the rat remnant kidney model of chronic kidney disease". Public Library of Science One 11.1 (2016): e0144640.

16. Layton AT., et al. "Modeling oxygen consumption in the proximal tubule: effects of NHE and SGLT2 inhibition". American Journal of Physiology Renal Physiology 308.12 (2015): F1343F1357.

17. Sano M., et al. "Increased hematocrit during sodium-glucose cotransporter 2 inhibitor thrapy indicates recovery of tubulointerstitial function in diabetic kidneys". Journal of Clinical Medicine Research 8.12 (2016): 844-847.
18. Aoki Y., et al. "Sodium-glucose co-transporter 2 inhibitors could improve the bioavailability of vitamin $\mathrm{C}$ at the kidney in diabetes treatment". Cellular and Molecular Medicine 3.2 (2017): 7-8.

19. Chiasson JL., et al. "Acarbose treatment and the risk of cardiovascular disease and hypertension in patients with impaired glucose tolerance: the STOP-NIDDM trial". The Journal of the American Medical Association 290.4 (2003): 486-494.

20. Scholl-Buergi S., et al. "Long-term outcome of renal glucosuria type 0: the original patient and his natural history". Nephrology Dialysis Transplantation 19.9 (2004): 2394-2396.

21. Calado J., et al. "Twenty-one additional cases of familial renal glucosuria: absence of genetic heterogeneity, high prevalence of private mutations and further evidence of volume depletion". Nephrology Dialysis Transplantation 23.12 (2008): 3874-3879.

22. Gann D., et al. "A low carbohydrate diet effective in the treatment of obese or diabetic patients with chronic kidney disease". Journal of Diabetes, Metabolic Disorders and Control 2.2 (2015): 54-57.

23. Praga M., et al. "The fatty kidney: obesity and renal disease". Nephron 136.4 (2017): 273-276.

24. Orlando A., et al. "Hypertension in children: role of obesity, simple carbohydrates, and uric acid". Frontiers in Public Health 6 (2018): 129.

25. Asghari G., et al. "Dietary pattern and incidence of chronic kidney disease among adults: a population-based study". Nutrition and Metabolism 15 (2018): 88.

\section{Volume 3 Issue 8 August 2019 (C) All rights are reserved by Yuji Aoki.}

